Nonlinear Processes in Geophysics (2002) 9: 149-162

Nonlinear Processes

in Geophysics

(C)European Geophysical Society 2002

\title{
Scaling and singularity characteristics of solar wind and magnetospheric fluctuations
}

\author{
Z. Vörös ${ }^{1}$, D. Jankovičová ${ }^{1}$, and P. Kovács ${ }^{2}$ \\ ${ }^{1}$ Geomagnetic Observatory Hurbanovo, Geophysical Institute SAS, Slovak Republic \\ ${ }^{2}$ Eötvös Loránd Geophysical Institute, Hungary
}

Received: 13 July 2001 - Revised: 23 November 2001 - Accepted: 18 January 2002

\begin{abstract}
Preliminary results are presented which suggest that scaling and singularity characteristics of solar wind and ground-based magnetic fluctuations appear to be a significant component in the solar wind-magnetosphere interaction processes. Of key importance is the intermittence of the "magnetic turbulence" as seen in ground-based and solar wind magnetic data. The methods used in this paper (estimation of flatness and multifractal spectra) are commonly used in the studies of fluid or MHD turbulence. The results show that single observatory characteristics of magnetic fluctuations are different from those of the multi-observatory $A E$-index. In both data sets, however, the influence of the solar wind fluctuations is recognizable. The correlation between the scaling/singularity features of solar wind magnetic fluctuations and the corresponding geomagnetic response is demonstrated in a number of cases. The results are also discussed in terms of patchy reconnection processes in the magnetopause and forced and/or self-organized criticality (F/SOC) of internal magnetosphere dynamics.
\end{abstract}

\section{Introduction}

According to recent knowledge magnetospheric plasma fluctuations may be explained in terms of multiscale intermittent models, including nonlinear self-organization processes (forced or not) near criticality (F/SOC) (Chang, 1992, 1999; Chapman et al., 1998; Takalo et al., 1999; Klimas et al., 2000). The basic physical concepts and the corresponding path-integral and renormalization group formalisms of nonlinear self-organization of magnetospheric processes are described in Chang (1999). These models predict multifractality, non-Gaussian, power-law distributions for certain measurable physical quantities and associated powerlaw scalings for power spectra. In fact, several experimental studies seem to support the theoretical predictions quite well (Consolini et al., 1996; Milovanov et al., 1996; Consolini, 1997; Borovsky et al., 1997; Consolini and De Michelis, 1998; Vörös, 1998; Uritsky and Pudovkin, 1998; Con- solini and Lui, 1999; Chapman et al., 1999; Freeman et al., 2000a,b; Sharma et al., 2001). Scale-free power spectra of magnetospheric fluctuations, however, admit several possible explanations other than F/SOC. Watkins et al. (2001a) compared linear noisy, low-dimensional nonlinear, stochastic fractional Brownian motion ( $\mathrm{fBm}$ ) and SOC models of magnetospheric fluctuations to examine their scaling and predictabilitity properties. They concluded that the SOC model is of particular interest to magnetospheric physics due to its robustness in explaining scalings under the wide range of activity levels exhibited by the magnetosphere and the solar wind. On the other hand, weakly nonlinear models with fBm type noise added could explain the scaling and predictabitity properties of magnetospheric fluctuations equally well. Geomagnetic fluctuations on the time scale of substorms and storms certainly appear to be a compound mixture of multiscale magnetospheric processes, including the component represented by intermittent solar wind fluctuations (Chang, 1999; Daglis et al., 1999). Spectral methods that make a distinction between these constituents may be queried to a certain extent mainly because the information on nonlinear multiscale structures is partially hidden for second order statistics. For example, Tsurutani et al. (1990), using the $A E$-index time series, have shown that the power spectrum of the $A E$ data exhibits two different power-law scalings divided by a spectral break at the frequency range about $1 /(5 \mathrm{~h})$. While the higher frequency part was thought to be more intrinsic to the magnetosphere, the lower frequency part was attributed to the influence of solar wind. Vörös (2000) has shown that similar results can also be obtained by means of a multifractal technique, which makes it also possible, however, to reveal additional information on singularity distributions of high-latitude geomagnetic fluctuations (see later) that are not evident from spectral studies. Among other things, the results of multifractal analysis show that the local singularity (Hölder) exponents are time dependent and the previously proposed $\mathrm{fBm}$ type or bicolored noise model (Takalo et al., 1993) of geomagnetic fluctuations is not relevant. Moreover, for a range of singularity exponents, a multi- 


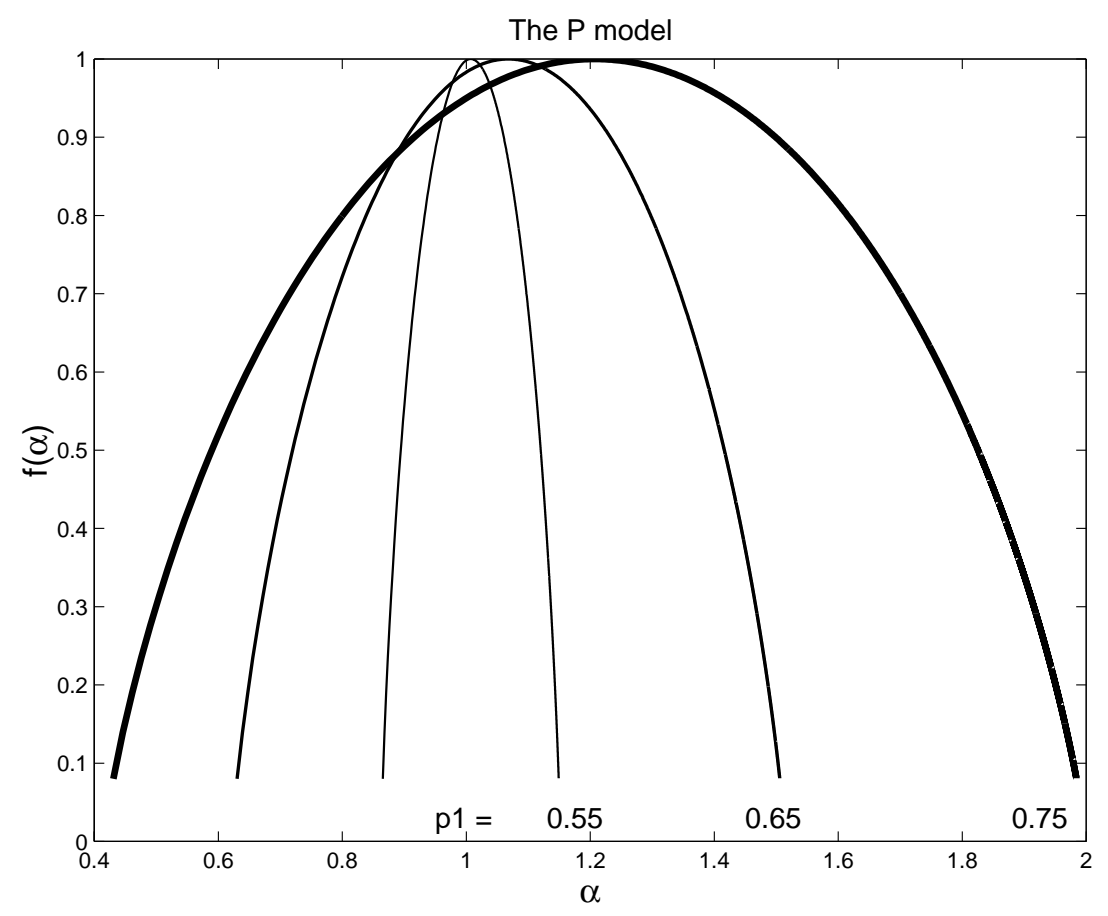

Fig. 1. The $P$-model for three different values of parameter $p_{1}$. plicative cascade model, (the $P$-model) describes quite well the observed singularity distribution of geomagnetic fluctuations. Out of this range, however, significant deviations from a multiplicative model appear. The $P$-model describes energy cascade processes in turbulent flows. The largest turbulent eddy is assumed to be built up by a specific energy flux per unit length. Then a scale-independent space-averaged cascade-rate is considered and the flux density is transferred to the two smaller eddies with the same length but different flux probabilities $p_{1}$ and $p_{2}$. This process with randomly distributed $p_{1}$ and $p_{2}\left(p_{1}+p_{2}=1\right)$ is repeated towards smaller and smaller scales. The energy transfer rate is homogeneous for $p=p_{1}=p_{2}=0.5$, while $p_{1}>0.5$ corresponds to an intermittent flow. Figure 1 shows how the intermittence increases with increasing parameter $p_{1}$ (Tu et al., 1996) in $\alpha, f(\alpha)$ plane (Halsey et al., 1986). The larger the spread is of $\alpha$ values around right-shifted $\bar{\alpha}$, the more intermittent the field. In spite of the simplicity of the outlined cascade model, its relevance for the description of intermittence effects in magnetospheric fluctuations may lead to the assumption that turbulence rather than F/SOC models fit the observed statistics better. Some results on statistical distribution of internal time periods between bursty geomagnetic events (waiting times) (Kovács et al., 2001) seem to support this assumption. SOC models (at least the original Bak et al. (1987) model) are expected to display an exponential waiting time distribution. Geomagnetic data, however, display a well-defined power-law waiting time distribution. It was pointed out by Boffetta et al. (1999) that the observed powerlaw waiting time statistics in solar flares appears to be well explained by MHD shell models of turbulence. Similar results were presented by Spada et al. (2001), who analysed density fluctuations in a magnetically confined plasma system and found that waiting time statistics are in contrast with the predictions of an SOC system. An opposite view was presented by Freeman et al. (2000b), who conjectured that a wider class of running sandpile models (Hwa and Kardar, 1992) could exhibit power-law behaviour in the probability density functions of waiting times. Watkins et al. (2001b) determined that the PDFs for burst durations and waiting times in a reduced MHD simulation follow power laws that are not sufficient to distinguish between turbulence, SOClike models and colored noise sources. Boffetta et al. (1999) and Antoni et al. (2001) also argued that SOC models represent self-similar, fractal phenomena. Geomagnetic fluctuations exhibit clear multifractal scaling (Consolini et al., 1996; Vörös, 2000), which seems again to contradict the SOC concepts. Georgoulis et al. (1995), however, demonstrated that the SOC state displayed by their cellular automaton model of isotropic and anisotropic energy avalanches has multifractal and multiscaling characteristics, rather than single power-law scalings, and this feature was even enhanced by considering extended instability criteria. Similar SOC models of solar flares also exhibit multifractal and multiscaling characteristics (Vlahos et al., 1995). Recent works (Vassiliadis et al., 1998; Isliker et al., 1998, 2000; Georgoulis et al., 2001; Uritsky et al., 2001b) underline the proximity between the SOC rules and laws of MHD in space physics systems, which are long known to exhibit turbulent behaviour (Georgoulis, personal communication, 2001). More realistic F/SOC models, which include, for example, less artificial feedback mechanisms, a wide variety of drivings, interacting avalanches, SOC in continuum physical systems (Lu, 1995), etc., may further establish the proximity with turbulence. As pointed 
out by Uritsky et al. (2001b) the concept of SOC in a continuum limit (fluid or MHD limit) is essentially unexplored. Klimas et al. (2000) proposed a simplified Earth's magnetotail, current sheet model based on the continuum SOC model of $\mathrm{Lu}$ (1995). The continuum $\mathrm{Lu}$ model in a magnetic field reversal configuration can evolve into SOC due to localized rapid magnetic field annihilation within the field reversal region. In the same time, the plasma sheet is dominated by strong turbulence which keeps the system near criticality and produces a predictable quasi-periodic loading-unloading cycle of coherent global substorm activity (Klimas et al., 2000). In this model, turbulence, SOC states and coherent global modes coexist within the Earth's magnetotail on different scales. As a result, the observed ground-based and satellite time series contain a mix of fluctuations of different physical origin. Angelopoulos et al. (1999) further argued that the presence of intermittent turbulence in the Earth's magnetotail may alter the conductivity and the mass/momentum diffusion properties across the plasma sheet and may permit cross-scale coupling processes that also play an important role in the establishment of SOC state.

In this paper, no attempt will be made to participate in the theoretical debate on SOC or turbulence. However, having a pragmatic view, our opinion is that measures of intermittence or characteristic descriptors of cascade processes, commonly used in turbulence studies, like the flatness and multifractal spectra, could be applied in solar wind-magnetosphere interaction studies for further comparison of basic characteristics of intermittent fluctuations in the solar wind and within the magnetosphere. We assume this aproach might be useful by providing experimental information on such characteristics of fluctuations that are not accessible for spectral studies or second order statistics. This assumption was already investigated in Vörös (1998, 2000); Vörös and Kovács (2001); Kovács et al. (2001). The preliminary results allow to make a working hypothesis such that intermittence, scaling, or rapid changes, singularities represent an essential piece of information regarding the effectiveness of solar wind-magnetosphere coupling not considered enough hitherto. In order to go deeper, we analyse different geomagnetic and solar wind data sets and make a comparison between various magnetic activity levels considering time scales of geomagnetic storms (from hours to days), substorms (from half an hour to a few hours) or less.

The main goal of this comparative study is to contribute to the understanding of solar wind-magnetosphere interaction processes on the basis of characteristic scaling/singularity features of the considered time series. We recall that solar wind (SW) fluctuations are strongly intermittent (Burlaga, 1991) that is energy at a given scale is not homogeneously distributed in space and/or time. Several studies on characteristic probability distribution functions (PDF) of increments of SW parameters (magnetic field, velocity, temperature, Elsässer variable, etc.) (Marsch and Tu, 1994; SorrisoValvo et al., 1999) and on the multifractal structure of SW fluctuations (Burlaga, 1992; Carbone, 1994; Marsch et al., 1996; Tu et al., 1996) support this assumption. Moreover,
Veltri and Mangeney (1999) and Bruno et al. (1999) have shown that there is a direct link between intermittence and the presence of SW structures (Alfvénic, magnetic fluctuations, discontinuities) across which the magnetic field magnitude changes. Also, the high frequency (small-scale) fluctuations of the southward component of the interplanetary magnetic field have a different spectral scaling exponent than the one exhibited by geomagnetic $A E$-index fluctuations. On larger scales the corresponding spectra are similar (Tsurutani et al., 1990). A comparative study of dynamical critical scalings in the auroral electrojet $(A E)$ index versus solar wind fluctuations confirmed that for times shorter than $3.5 \mathrm{~h}$ (higher frequencies) the $A E$-index fluctuations are of internal magnetospheric origin (Uritsky et al., 2001a).

\section{Data analysis methods}

As usual, we introduce the concept of scale $(\tau)$ through the difference

$\delta X(t, \tau)=X(t+\tau)-X(t)$,

where $X(t)$ is the time series under consideration. Marsch and Tu (1994) have shown that the PDFs of the increments $\delta X$ ( $X$ - SW parameters) exhibit strong deviations from Gaussianity, especially at smaller scales and the effect is due to intermittence of SW fluctuations. To quantify the degree of deviation from the Gaussian distribution, i.e. the level of intermittence at different scales, we compute the flatness defined by

$$
F=\frac{\left\langle\delta X(t, \tau)^{4}\right\rangle}{\left\langle\delta X(t, \tau)^{2}\right\rangle^{2}} .
$$

The flatness of a normally distributed signal is equal to 3 . Adding intermittent fluctuations to an originally Gaussian signal implies the spreading of its PDF, and consequently, the increase of its flatness from the original value of 3 .

Non-homogeneous/intermittent distributions in space/time may also appear as asymptotically singular and can be characterized locally at the point $t_{i}$ by the singularity (Hölder) exponents $\alpha$ (Véhel, 1996; Véhel and Vojak, 1998; Riedi, 1995; Canus, 1998) as

$\alpha_{n}^{k}\left(t_{i}\right)=\lim _{n \rightarrow \infty} \frac{-\log \mu\left(I_{n}^{k}\left(t_{i}\right)\right)}{n}$,

where $\mu$ is a measure constructed from a time series. The procedure involves the computation of the energy content of the signal difference (Eq. 1) by taking its squared value. The measure at a point $t_{i}$ is given by $\delta X^{2}\left(t_{i}, \tau\right) / \sum_{i} \delta X^{2}\left(t_{i}, \tau\right)$. To analyse the distribution of singularity exponents (Eq. 3) a sequence of partitions $P$ is introduced so that (Véhel, 1996)

$$
\begin{gathered}
P_{n}=\left\{I_{n}^{k}\right\} ; \quad 0 \leq k<2^{n}-1 \\
\left.I_{n}^{k}=<k 2^{-n},(k+1) 2^{-n}\right),
\end{gathered}
$$

where $I_{n}^{k}$ is the interval containing $t$ and the resolution is set by $n$. The quantity of interest is the so-called large deviation 
singularity spectrum, $f(\alpha)$, which represents a rate function measuring the deviation of the observed $\alpha$ from the expected value $\bar{\alpha}$. The rate function, $f(\alpha)$, can be estimated through (Véhel, 1996):

$f(\alpha)=\lim _{n \rightarrow \infty} \frac{N_{n}(\alpha)}{n}$,

where $N_{n}(\alpha)$ is the observed number of coarse grain Hölder exponents. Usually "histogram methods" for estimation of $f(\alpha)$ are used. In that case, the number of those intervals $I_{n}^{k}$ for which $\alpha_{n}^{k}$ falls in a box between $\alpha_{\min }$ and $\alpha_{\max }$ is computed and $f(\alpha)$ is found by a regression. It yields satisfactory results for pure multiplicative processes, but fails to describe non-pure or compound processes when $f(\alpha)$ is not a concave function. To overcome this difficulty, the so-called double kernel method was proposed (Véhel, 1996; Véhel and Vojak, 1998) realizing that $N_{n}(\alpha)$ may be written as a convolution of the density of the $\alpha_{n}^{k}$ s and a compactly supported kernel. This method allows one to estimate non-concave rate functions and we are going to show that this property may be properly used for the characterization of fluctuation processes in near-Earth space. In this paper, the estimations of $f(\alpha)$ spectra were realized by using the FRACLAB package developed at the Institut National de Recherche en Informatique, Le Chesnay, France.

\section{Ground based data}

In order to study the basic characteristics of auroral zone geomagnetic fluctuations, we analyse geomagnetic $H$ component 1-min mean data from the polar cap observatory, THULE (THL: $77.47^{\circ} \mathrm{N}, 290.77^{\circ} \mathrm{E}$ ), geomagnetic $X$ component 1-min mean data from the high-latitude observatory NARSSARSSUAQ (NAQ: $61.16^{\circ} \mathrm{N}, 314.57^{\circ} \mathrm{E}$ ) and 1min mean auroral electrojet $(A E)$ index time series, all from 1991-1992.

As known, the $A E$-index was introduced by Davis and Sugiura (1966) to describe the global activity of the auroral zone electric currents and is derived, after the substraction of base line values, from evaluation of the variations measured at 12 stations located near the northern auroral zone. There exist a large number of physical mechanisms which couple the auroral zone processes with those within the magnetospheric tail or in the SW. Recently, intermittent energy transport in the magnetotail, the so-called bursty bulk flow events (BBF) came into the limelight of the magnetosphere research (Baumjohann et al., 1990; Angelopoulos et al., 1992). From this point of view the understanding of the response of auroral zone currents or dissipation fields to the time-varying magnetotail dynamics seems to be important. A full understanding was not achieved regarding the nature and origin of the related magnetic fluctuations. It was already partly explained in the Introduction that it is related to the paradigms of F/SOC versus turbulence. We mention here some other open questions. For example, it was shown that the burst lifetime distributions of some SW parameters are also of powerlaw form, which might be a signature of SOC or turbulence regimes in the SW (Freeman et al., 2000a; Kovács and Vörös, 2001). Therefore, it is assumed that the scale free property of the $A E$-index may arise from the $\mathrm{SW}$ input or at least the internal dynamics of the magnetosphere may be masked by the scale free properties of the SW driver (Freeman et al., 2000a). Again we are reminded, however, of the very limitations of second order statistics in the interpretation of the observed scalings. Another measure of the auroral zone dissipation fields is represented by polar optical activity within UVI bands. Lui et al. (2000) have examined the blobs of brightness as a proxy for BBF events. It was found that the non-substorm "internal" events have a power-law distribution whereas the system wide events, such as substorms, exhibit, in addition to a scale free region, a "bump", corresponding to a mean value in substorm breakups. A somewhat opposite view was presented by Consolini and De Michelis (1998), who analysed $A E$-index fluctuations on time scales of 1-120 min both in quiet (laminar phase) and disturbed periods (turbulent phase). They found that in both phases the intermittence at different time scales rescales in the same way and the non-Gaussian character of the PDFs seems to be due to the same physical processes.

Here we pose the question again about the scaling and singularity properties of the $A E$-index, compared to the similar characteristics of geomagnetic data from the two observatories THL and NAQ. Since the $A E$-index is derived from geomagnetic variations in the horizontal component observed at selected observatories along the auroral zone in the Northern Hemisphere, we expect that the scale $\tau$ (Eq. 1) cannot be precisely defined. The geographic distance between observatories through Taylor's hypothesis already introduces some effective time shift (scale, $\tau$ ), though the application of Taylor's hypothesis within the magnetosphere is limited (Dudok de Wit and Krasnoselskikh, 1996). Besides, from the recordings of auroral stations, the greatest (upper envelope) and smallest (lower envelope) values are taken at intervals of one minute and their difference defines the $A E$ index. As far as the contributing observatory, which gives the lower/upper envelope changes during the times, and the fact that the fluctuations with values between the upper and lower envelopes are not at all taken into account (smoothing), the $A E$-index appears to be a measure of auroral zone processes with mixed scales. This is certainly not an advantage when a multiscale analysis of the $A E$-index time series is performed. This fact is usually neglected in the related literature. A less sophisticated way is to take data from a single observatory, but this may have some other drawbacks because significant distant disturbance events can be missed. Also, some BBFs with short duration may remain undetected on the ground due to their localized nature (Daglis et al., 1999). Nevertheless, a comparison of fluctuations of the "multi-observatory measure" (MOM: $A E$-index) and of the "single observatory measure" (SOM: THL geomagnetic field $H$-component and NAQ geomagnetic field $X$-component) may be instructive.

Figure 2 shows the PDFs from normalized increments $\delta X$ (Eq. 1) of the SOM (Figs. 1a and b) and MOM (Fig. 1b) data $(X \equiv X$ (NAQ), $H(\mathrm{THL})$ and $A E$, respectively). Cal- 

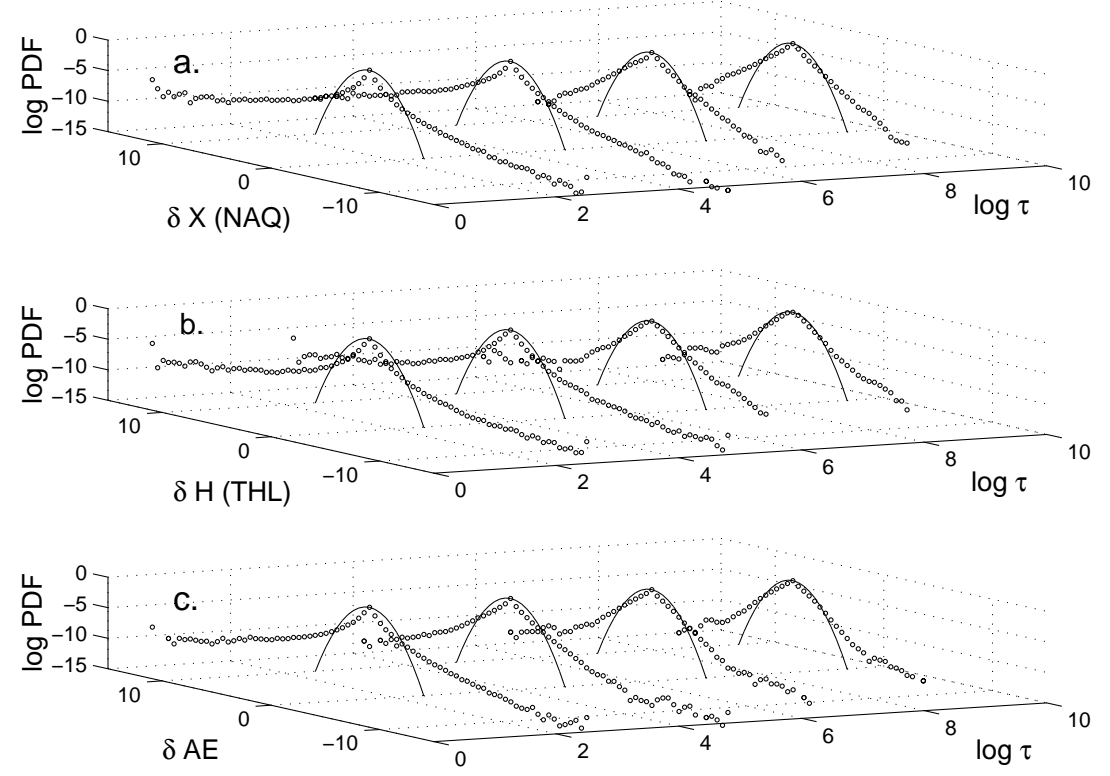

Fig. 2. Probability distribution functions computed for: (a) NARSSARSSUAQ $X$-component geomagnetic field; (b) THULE $H$ component geomagnetic field; (c) $A E$-index data. The time scales are $\tau=5,50,500,5000 \mathrm{~min}$. Continuous lines refer to Gaussian distribution, while the "o" markers correspond to the analysed data.

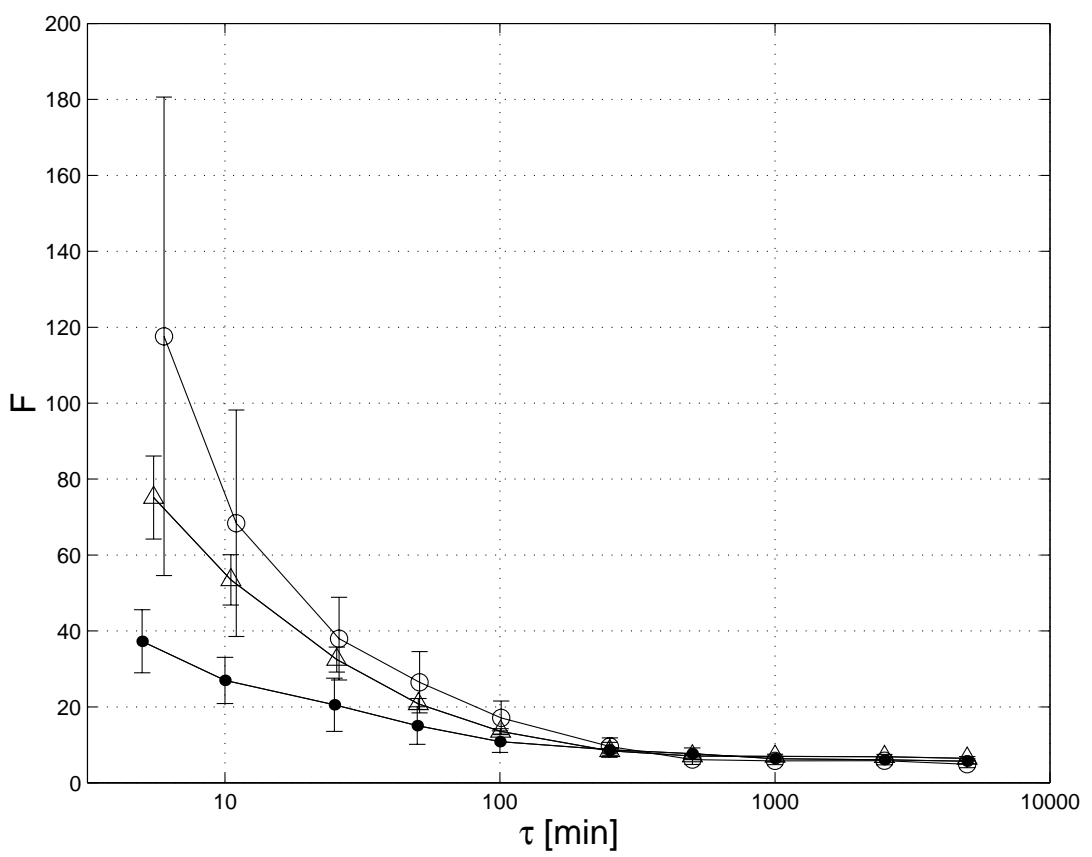

Fig. 3. The flatness as a function of scale $(\tau \in(5,5000) \mathrm{min})$ estimated for NARSSARSSUAQ $(\triangle)$, THULE data (o) and $A E$-index $(\bullet)$.

culations have been made for $\tau=5,50,500,5000 \mathrm{~min}$ and 1 min mean data were considered from the years 1991-1992. As can be seen, the distributions in all cases change with $\tau$, and for smaller values of $\tau$, significant deviations from the normal distribution occur. The tails of the distributions reduce with increasing scale parameter as a consequence of the decrease in the probability of coherent fluctuations between points separated by increasing distance. The difference between the MOM and SOM is more clear if the flatness $(F)$ of the corresponding distributions is compared (Eq. 2). Figure 3 shows how the flatness evolves with increasing $\tau$ ( $\tau$ $\in(5,5000) \mathrm{min})$. The error bars correspond to the standard deviations (std) computed from the time series divided into several parts. For small scales, for example, $\tau$ less than a few tens of minutes, the $A E$-index (MOM) exhibits smaller deviations from the normal distribution than NAQ (SOM). $F(\mathrm{NAQ})$ reaches the level of $F_{\max }(A E, \tau=5)$ only at the value $\tau \sim 30 \mathrm{~min}$, which roughly may be considered as the effective time shift introduced by the method of derivation of the $A E$-index. THL (polar cap observatory) data essentially show the same behaviour as NAQ, but the stds are larger. Therefore, we conjecture that MOMs (multi-observatory geomagnetic indices), due to smoothing and scale mixing effects, lead to an underestimation of the intermittence on 

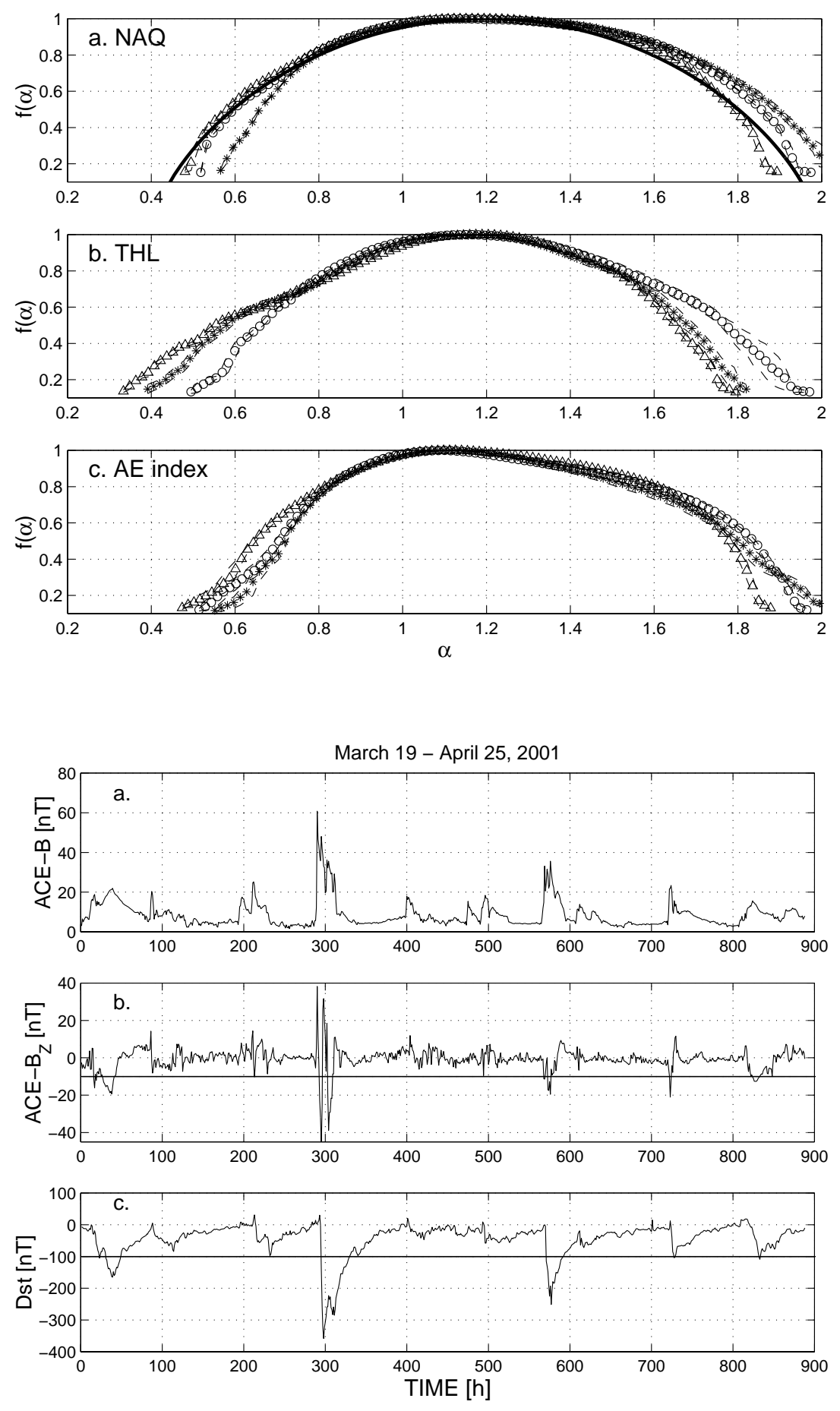

Fig. 4. Dependence of singularity rate functions on scale; $\tau=5(\triangle) ; 50(*) ; 500(\mathrm{o}) \mathrm{min}$. (a) NARSSARSSUAQ data, (b) THULE data; (c) $A E$-index. Thick line in Fig. 4a corresponds to $P$-model fit.
Fig. 5. An extremely disturbed period as seen in 1-h mean (a) ACE IMF magnitude, (b) ACE IMF $B_{z}$ component and (c) $D_{s t}$-index data plots. small scales. Henceforth, we will show only the dependence of the flatness on scale parameter $\tau$.

Let us consider now the singularity distributions $f(\alpha)$ (Eqs. 3 and 4) for the same data sets as above. To see better how the $f(\alpha)$ rate functions evolve with $\tau$, all the curves are depicted at the same plane $(\alpha, f(\alpha))$ in Fig. 4. Proper symbols are introduced for the scales $\tau=10,50,500 \mathrm{~min}$ $(\triangle, *, o)$ and the dashed lines correspond to the errors estimated by changing the resolution in Eqs. (3) and (4). (No averaging needs to be done when estimating $f(\alpha)$ (Véhel, 1996), so the spectrum may be evaluated at only one resolution. However, to show that the estimations are consistent, all the spectra were computed using 15 different resolutions). Again, there are several differences between the SOM and MOM data. Figure $4 \mathrm{a}$ shows the singularity spectra for NAQ observatory data. On the considered scales, the shape of the curves is almost parabolic, close to the $P$-model fit (thick curve), with $p_{1} \sim 0.745$. The best correspondence 
is achieved for the smallest value of $\tau=10 \mathrm{~min}$ (depicted by symbol $\Delta$ on Fig. 4a). It means that in case of auroral zone SOM fluctuations and especially on small scales, the deviations from the Gaussian distribution (Fig. 3) can be explained by a simple cascade model. Though the phenomenology of turbulent cascades in fluid flows is more complex than the simple $P$-model fit in Fig. 4a would lead us to indicate, the 1D cascade model roughly describes how the auroral zone SOM fluctuations become more and more intermittent at smaller and smaller scales. The spectra for polar cap (THL observatory) SOM (Fig. 4b) and $A E$-index MOM (Fig. 4c) have a more pronounced non-parabolic shape, indicating the presence of compound processes. At small scales $(\tau=5,50 \mathrm{~min})$, THL observatory fluctuations contain stronger singularities due to the extension of the rate functions' left wings to the smaller (more singular) values of $\alpha$. For $\tau=500 \mathrm{~min}$, however, the right wing evolves to a less singular value, which may be related to the $\mathrm{SW}$ influence (Vörös, 2000). This effect is less visible, but is still present in auroral zone SOM data (Fig. 4a). The MOM $A E$-index $f(\alpha)$ spectra hardly change with $\tau$. We conjecture that this is the result of the method of derivation of the $A E$-index, resulting in smoothing and scale mixing effects.

In all cases, the deviations of singularity spectra from the parabolic shape may be indicative of the phenomenon of phase transition. Namely, at the $\alpha$ values, where the $f(\alpha)$ spectra are not in a parabolic shape, the major contributor to the observed singularities may change from one measure to another. As different physical processes may generate different measures (distributions), possible models with similar characteristics as the observed spectra may contain physical information on the contributing (e.g. SW or magnetospheric) sources.

\section{Satellite data}

The very advantage of the ground-based data is its availability for long periods of time. For a proper estimation of PDFs or singularity rate functions, long data sets are needed which is a requirement that is hardly ever fulfilled in the case of satellite data. Nevertheless, we expect to find out some interesting scaling/singularity features of interplanetary magnetic field (IMF) fluctuations proceeding in the same way as in the previous section. To this end, we analyse ACE and WIND IMF $B$ magnitude and $B_{z}$ component data, which are available with a time resolution of $16 \mathrm{~s}$ and $3 \mathrm{~s}$, respectively. SW velocity is not considered here because of too many gaps in the data. While the ACE satellite is continuously monitoring the SW at the $L_{1}$ point, WIND has a more complicated trajectory that also crosses the magnetosphere from time to time. For our analysis we have chosen time periods when WIND was also in the SW, and there were negligible data gaps in both cases (less than $1 \%$ of the total data lengths).

Another aim was to analyse "geoeffectively different" periods of IMF $B$ and $B_{z}$ magnitude fluctuations. Geoeffectiveness during the chosen periods was considered by exam- ining the geomagnetic $D_{s t}$ index, which is derived from the geomagnetic field $H$-component registrations of 4 observatories (Sugiura, 1964) and it aims at giving the effect of the magnetospheric ring currents. The chosen periods were classified as disturbed ones if geomagnetic fluctuations with a storm-index $D_{s t}$ of less than $-50 \mathrm{nT}$ occured several times within a considered interval. The limit of $D_{s t}<-100 \mathrm{nT}$, which corresponds to intense storms, was considered as well. We emphasize, however, that instead of a study of individual storms, the generic features of fluctuations on a given scale $\tau$ are investigated, but during longer periods of time. Essentially, 2-4 weeks of data with the considered time resolutions may already ensure sufficiently robust estimations of singularity spectra (Eq. 4). In this sense, several intense magnetic storms $D_{s t} \ll-50 \mathrm{nT}$ may occur during a strongly disturbed interval, where a less disturbed period contains less intense storms and an undisturbed period has only $D_{s t}>-50 \mathrm{nT}$. The limit of $-50 \mathrm{nT}$ was chosen on the basis of previous studies of magnetic storms (Taylor et al., 1996). Intense magnetic storms are characterised by $D_{s t}$ index $<-100 \mathrm{nT}$ (Gonzalez and Tsurutani, 1987). In this preliminary study of generic features of magnetic fluctuations, these limits may be considered as more or less adequate. We expect that this rough classification of the geomagnetic response allows us to identify characteristic scaling and singularity features of the corresponding IMF magnetic fluctuations that would be indicative for their geoeffectiveness.

There were 5 time periods and 6 data sets separated for our analysis (for one period there were both ACE and WIND data available). The disturbed periods are the following: (1) 19 March - 25 April 2001 (ACE); (2) 1 October - 30 November 2000 (ACE); (3) 9 April - 20 April 1997 (WIND). The undisturbed periods: (4) 18 November - 10 December 1998 (ACE); (5) 10 January - 29 January 1998 (ACE, WIND). For demonstration we show some of the data sets.

Figure 5 shows the first ACE data set from 19 March to 25 April 2001. For the sake of perspicuity, the time resolution is $1 \mathrm{~h}$ (the flatness and singularity spectra are computed from the time series with a time resolution of $16 \mathrm{~s}$ and $3 \mathrm{~s}$ ). During this extremely active period, intense magnetic storms $\left(D_{s t} \leq-100 \mathrm{nT}\right)$ occured several times (Fig. 5c). The limit of $-100 \mathrm{nT}$ is depicted by a thick line in Fig. 5c. Gonzalez and Tsurutani (1987) have shown that, the interplanetary causes of intense magnetic storms are long duration $(>3 \mathrm{~h}$ ), large and negative $(<-10 \mathrm{nT})$ IMF $B_{z}$ events, associated with interplanetary duskward electric fields $>5 \mathrm{mVm}^{-1}$. In Fig. 5b IMF $B_{z}$ is depicted, including a thick line indicating the level of $-10 \mathrm{nT}$. Comparison of Figs. $5 \mathrm{~b}$ and c shows an agreement with the above criteria, i.e. long duration negative IMF $B_{z}$ events occur together with intense magnetic storms. Figure $5 \mathrm{a}$ shows the variations of IMF $B$. It is visible that an intense magnetic storm occured at the end of the studied period, between $t=800$ and $900 \mathrm{~h}$ (Fig. $5 \mathrm{c}$ ). $B_{z}<10 \mathrm{nT}$ (Fig. 5b) and $B_{\max } \sim 17 \mathrm{nT}$ corresponded to this event. A similar enhancement of $B$ at $400 \mathrm{~h} \leq t<450 \mathrm{~h}$ appears in Fig. 5a having no intense storm response in $D_{s t}$, which can be explained by the corresponding IMF $B_{z}>-10 \mathrm{nT}$ in 

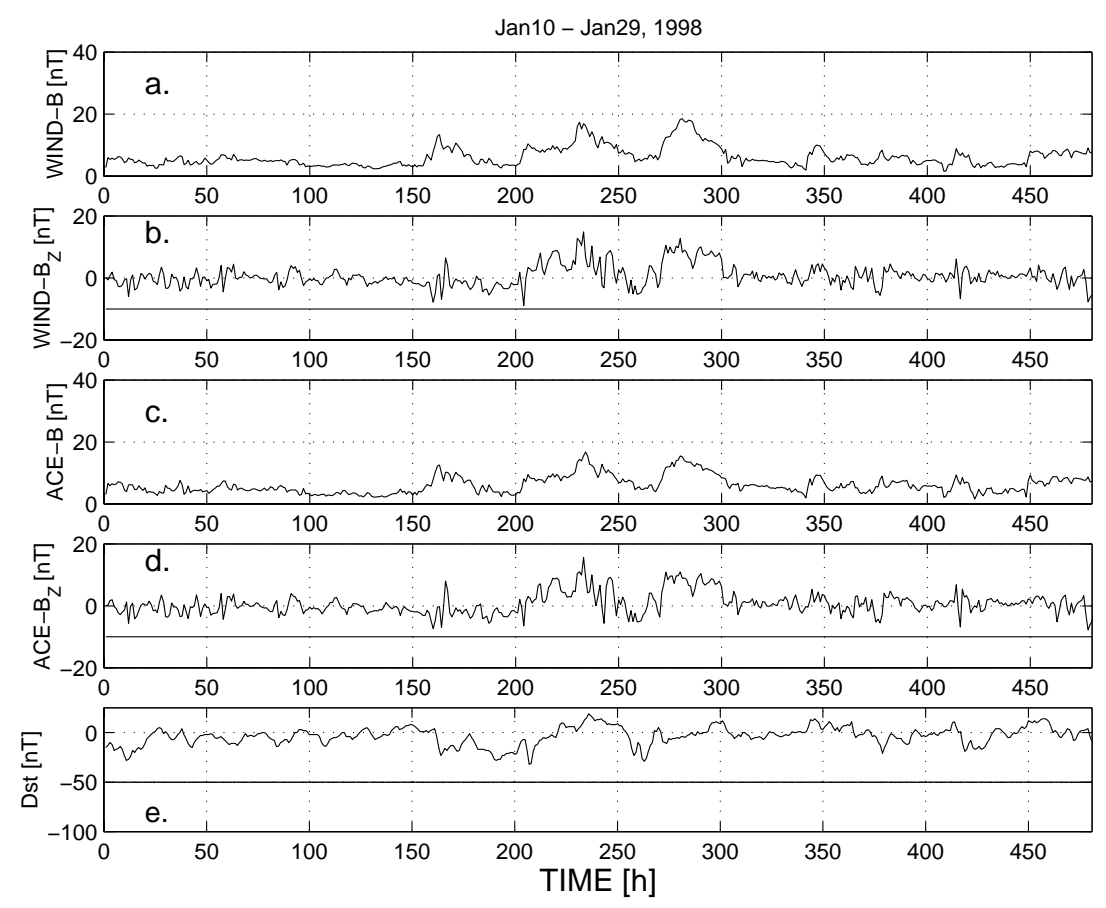

a. IMF-B
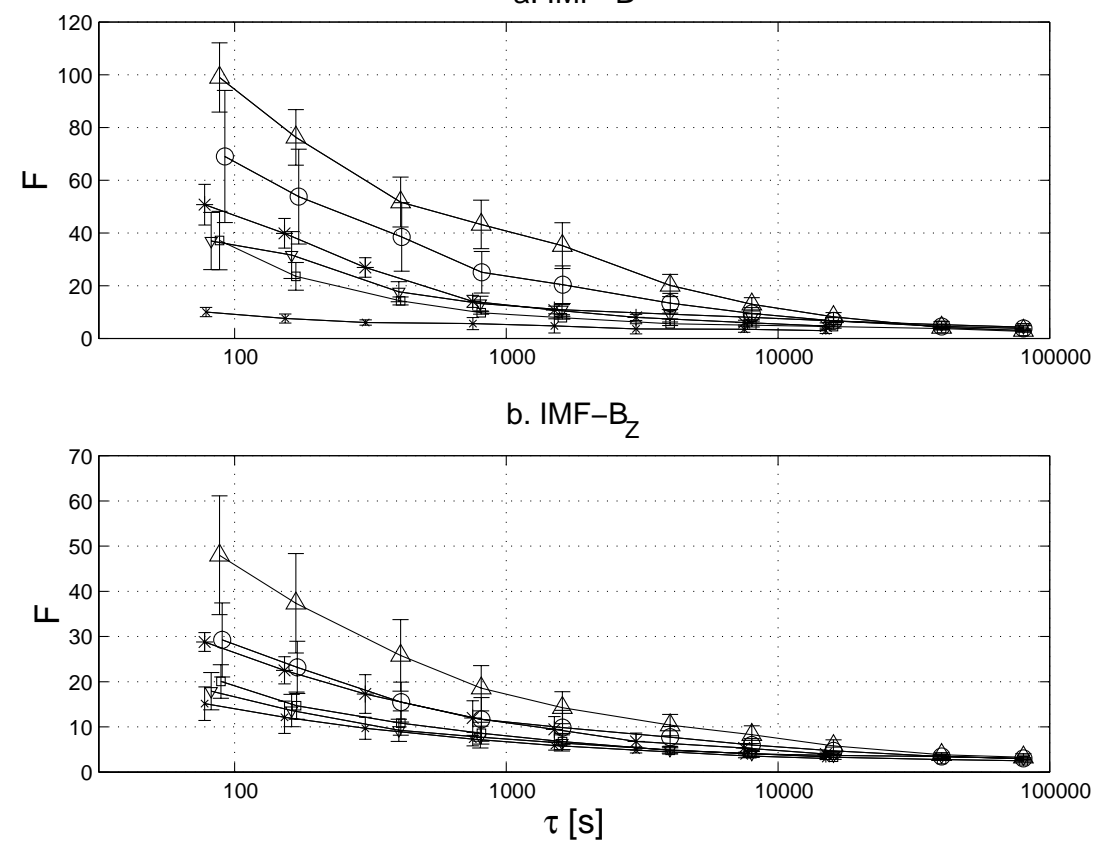

Fig. 6. Undisturbed period as seen in 1-h mean (a) IMF $B$ (WIND); (b) IMF $B_{z}$ (WIND); (c) IMF $B$ (ACE); (d) IMF $B_{z}$ (ACE) data plots and (e) the corresponding geomagnetic response represented by $D_{s t}$-index.

Fig. 7. The flatness as a function of scale $\left(\tau \in\left(16 * 5,16 * 10^{5}\right)\right.$ s) estimated for the periods: 19 March -25 April 2001, (ACE: $\triangle$ ); 1 October -30 November 2000, (ACE: o); 9 April 20 April 1997, (WIND: $*$ ); 18 November - 10 December 1998, (ACE: $\nabla) ; 10$ January - 29 January 1998, (WIND: $x$ ); 10 January - 29 January 1998, (ACE: $\diamond)$. Disturbed, i.e. geoeffective events, are depicted by larger marker sizes. (a) IMF $B$, (b) IMF $B_{z}$.

Fig. 5b.

Figure 6 shows an undisturbed period from 10 January to 29 January 1998. Again, IMF $B, B_{z}$ (WIND, ACE Figs. 6ad) and $D_{s t}$ index (Fig. 6e) are shown. It is visible that $D_{s t}>$ $-50 \mathrm{nT}$ and $B_{z}>-10 \mathrm{nT}$ everywhere.

Fluctuations of IMF and their geoeffectiveness were studied by a number of authors. McPherron et al. (1986) showed that substorms are frequently triggered by changes in the
IMF. Kamide (2001) proposed that the quasi-steady component of the interplanetary electric field is imporant in enhancing the ring current, while its fluctuations are responsible for initiating magnetospheric substorms. It is out of scope of this paper to analyse the influence of other interplanetary parameters (e.g. velocity, density, temperature, etc.) on storm/substorm activity (Daglis et al., 2001). Rather, we will concentrate on the level of intermittence of IMF fluctuations. 

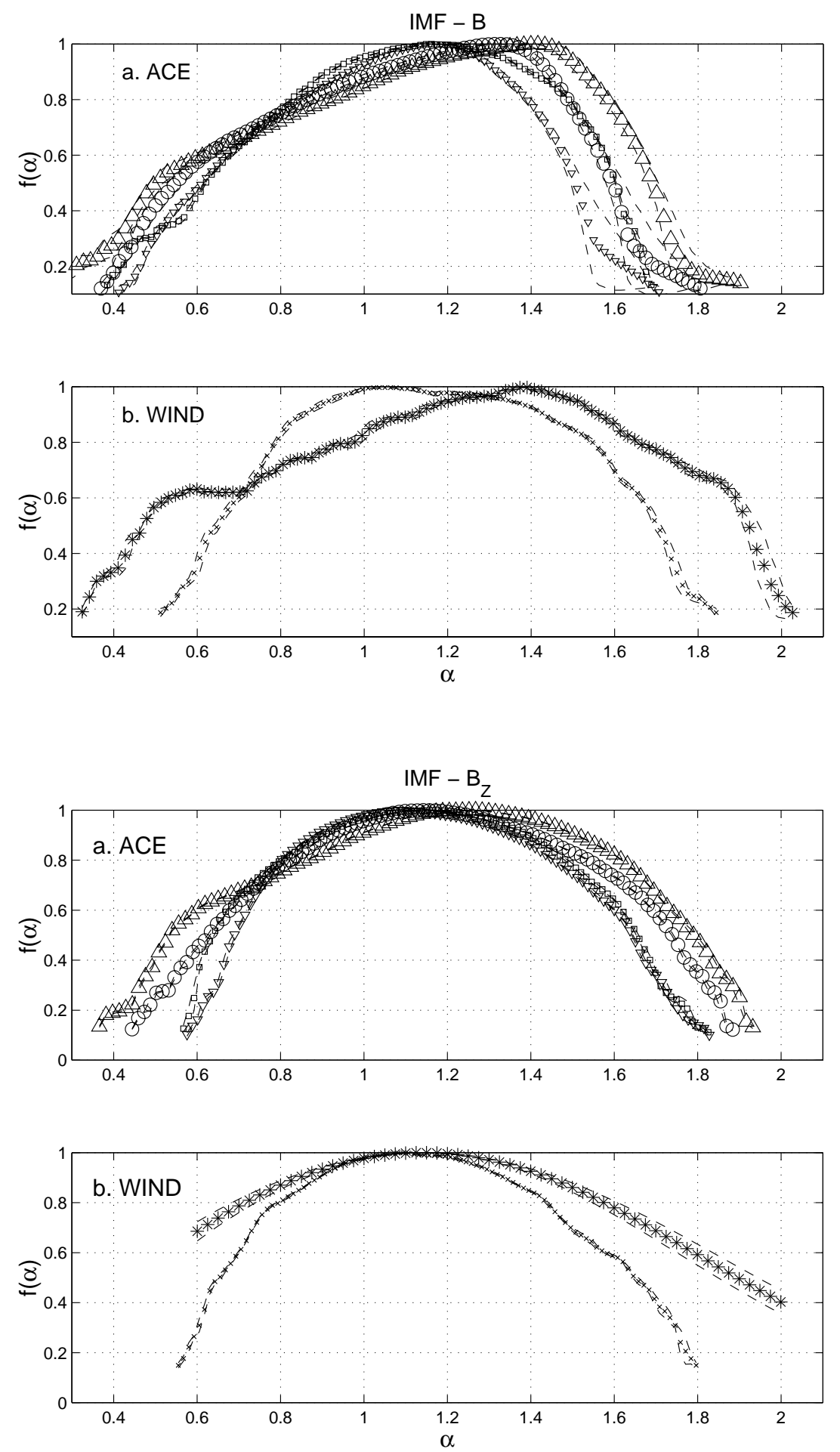

Fig. 8. Singularity spectra (rate functions) estimated for IMF $B$. The periods and data sets are depicted by the same marker types as in Fig. 7.
Fig. 9. Singularity spectra estimated for IMF $B_{z}$. The periods and data sets are depicted by the same marker types as in Fig. 7.
To this end, let us consider the flatnesses and the singularity rate functions for the disturbed and undisturbed periods of $1-5$. Deviations from the Gaussian distribution are larger in the case of disturbed events depicted by larger marker sizes in Fig. 7. The PDFs of the undisturbed events are also nonGaussian, but the flatnesses for a given scale are smaller than those for disturbed events, especially at scales $\tau<1000 \mathrm{~s}$.
Similar differences are present in singularity spectra computed for IMF $B$ fluctuations at the scales of $\tau=320 \mathrm{~s}$ (ACE) and $\tau=60 \mathrm{~s}$ (WIND), shown in Fig. 8. The same marker types were used as in Fig. 7. ACE and WIND data are depicted separately in Figs. 8a and b. The maxima of the singularity spectra of more disturbed periods have a tendency to shift to larger values of $\alpha$. Also, the spread of singulari- 

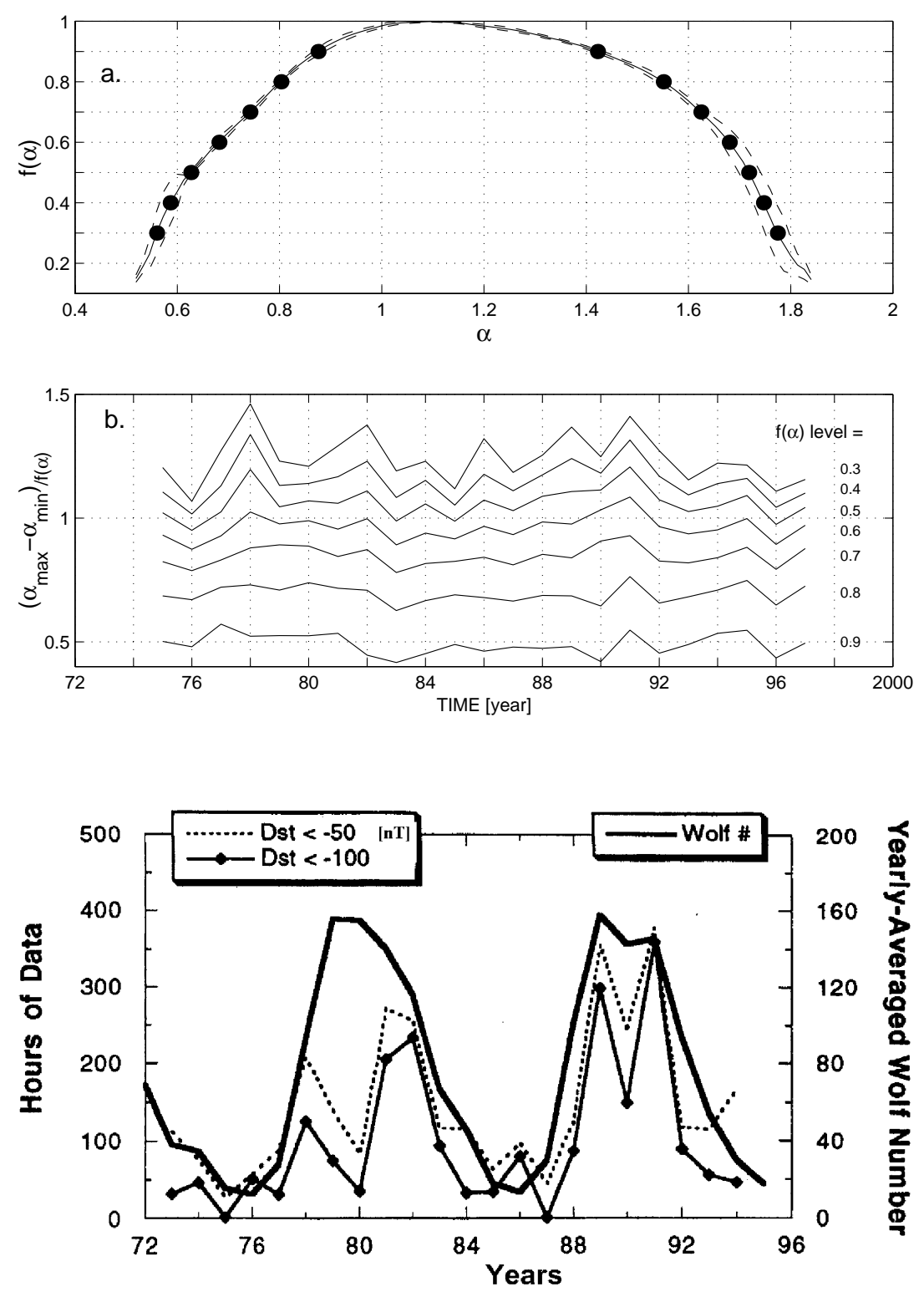

Fig. 10. Time variation of the shape of singularity spectra (a) filled circles show the points in which $\alpha_{\min }$ and $\alpha_{\max }$ values are computed; (b) $\alpha_{\max }-\alpha_{\min }$ values computed at different $f(\alpha)$ levels.
Fig. 11. Yearly averaged number of hours with $D_{s t}<-100 \mathrm{nT}(\diamond)$, and with $D_{s t}<-50 \mathrm{nT}(---)$. Yearly averaged sunspot number (thick line) is also shown. After Kamide et al. (1998). Reproduced by permission of American Geophysical Union. ties around the most probable $\alpha$ is wider for more disturbed cases, but it is the same behaviour as in the case of the simple $P$-model in Fig. 1, when intermittence is stronger and stronger for larger and larger values of $p_{1}$. The differences between the disturbed and undisturbed cases gradually cease for larger values of $\tau$ (not shown).

Figure 9 shows the singularity spectra computed for IMF $B_{z}$ fluctuations in the same way as previously stated. Obviously, the intermittent fluctuations of the IMF $B$ and $B_{z}$ fields exhibit very similar changes in their flatnesses and singularity spectra as the geoeffectivity level changes. For example, there is a clear difference between the introduced scaling and singularity characteristics (Fig. 5) for the disturbed period of 19 March - 25 April 2001 and for the undisturbed period of 10 January - 29 January 1998 (Fig. 6). It indicates that, in addition to known "geoeffective" SW parameters (e.g. southward component of IMF) or their combinations, small scale, rapid changes, singularities and non-Gaussian statistics of IMF fluctuations may play an important role in the SW-magnetosphere interaction processes.

Also, the question naturally arises as to what extent the magnetospheric response itself is influenced by small scale statistics of IMF fluctuations. Unfortunately, not all the geomagnetic data are available for the above analysed periods. It is possible to test, however, how the shape of the rate function changes if available data is considered. One Minute mean $H$-component geomagnetic data from the THL observatory is available from 1975 to 1996 . We computed the $f(\alpha)$ spectra for each year at the scale $\tau=50 \mathrm{~min}$ and analysed how their shapes change at the $f(\alpha)$ values $0.3-0.9$. At each level of $f(\alpha)$ the corresponding values of $\alpha_{\min }$ and $\alpha_{\max }$ were computed (filled circles in Fig. 10a). In Fig. 10b, the time evolution of the difference $\alpha_{\max }-\alpha_{\min }$ at a given $f(\alpha)$ level is depicted. The average standard deviation at 

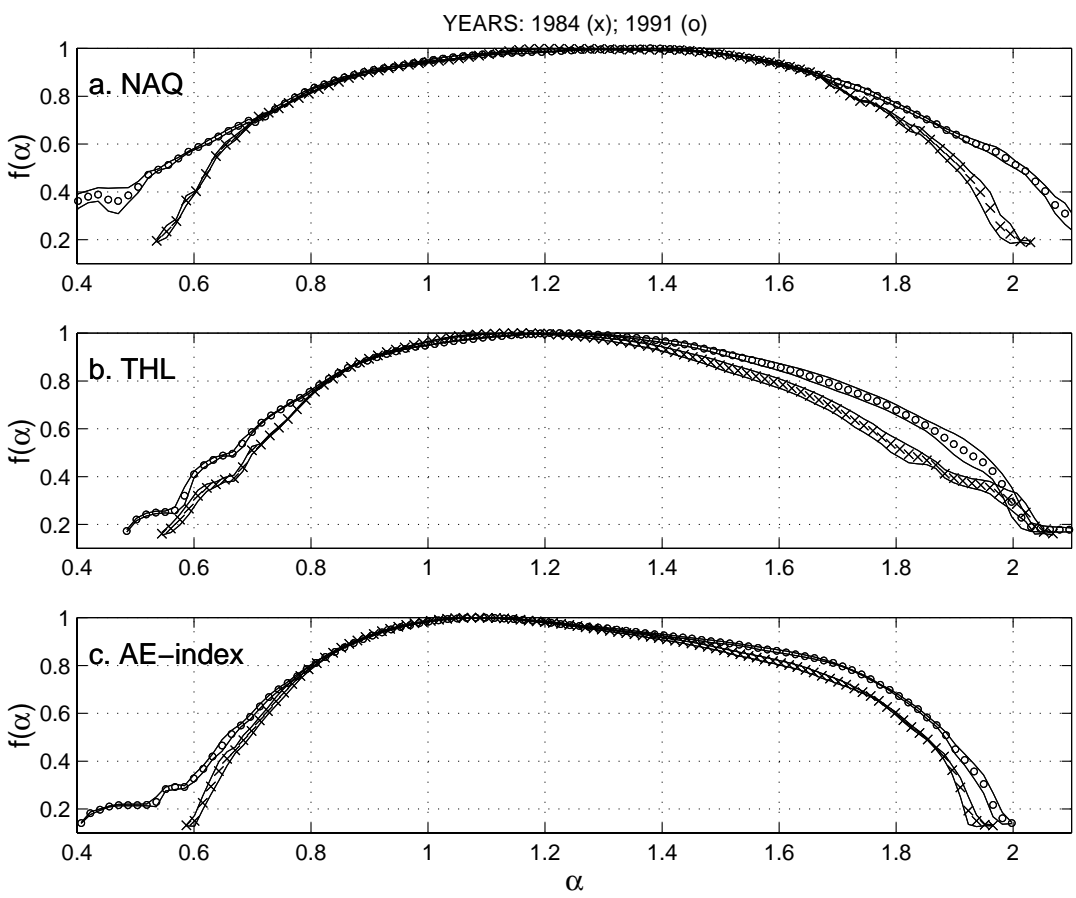

Fig. 12. Singularity spectra for (a) NARSSARSSUAQ data; (b) THULE data; (c) $A E$-index. Maximum of geomagnetic activity in 1991 (o); minimum of geomagnetic activity in $1984(x)$. $f(\alpha)=0.3$ is about 0.06 , while at $f(\alpha)=0.9$ is 0.02 . At $f(\alpha)=0.3-0.6$, the $\alpha_{\max }-\alpha_{\min }$ curves strongly fluctuate, indicating significant changes in the shape of $f(\alpha)$ spectra from 1975 to 1996 . Figure 11 shows the similar results of Kamide et al. (1998), however, obtained by a different method. Kamide et al. (1998) analysed the occurence of geomagnetic storms in comparison with the yearly averaged Wolf sunspot number. Figure 11 shows the yearly averaged number of hours with $D_{s t}$ less than $-100 \mathrm{nT}$ (solid line with filled diamonds), and with $D_{s t}$ less than $-50 \mathrm{nT}$ (divided by 5 , dashed line). Thick line corresponds to the yearly averaged sunspot number. It is visible that the maxima of geomagnetic activity and the maxima of the solar cycle do not coincide. During the declining phase of the solar cycle, coronal holes emerge from polar regions of the Sun that are continuous sources of fast-speed plasma, which cause a peak in recurrent geomagnetic storm activity (Kamide et al., 1998; Kamide, 2001). The similarity between the variability of rate function shapes for $f(\alpha)=0.3 \div 0.6$ (Fig. 10b) and the yearly averaged number of hours with prescribed $D_{s t}$ indices (Fig. 11) is remarkable. This correspondence also supports our working assumption that the shape of the $f(\alpha)$ rate function estimated using Eqs. (3) and (4) (Véhel, 1996; Véhel and Vojak, 1998) contains relevant physical information. We mention that the available $A E$-index and NAQ observatory data lead to the same results. On the other hand, however, at $f(\alpha)=0.7-0.9$, the variations of $\alpha_{\max }-\alpha_{\min }$ versus time are negligible (Fig. 10b).

On the basis of Figs. 10b and 11, years with extreme levels of geomagnetic response can be chosen and their corresponding singularity spectra can be recalculated. Figures $12 \mathrm{a}-\mathrm{c}$ show the SOM and MOM spectra estimated at $\tau=50 \mathrm{~min}$ for maximum (1991, symbol $o$ ) and minimum (1984, symbol $x$ ) years of geomagnetic activity. One can see that the characteristic asymmetric shape of $A E$-index spectra (Fig. 12c) in comparison with Fig. $4 \mathrm{c}$ is present henceforward, presumably caused by the influence of SW fluctuations. Noticeably, the maxima and the right wings of the more geoeffective SW IMF singularity distributions (Figs. 8 and 9) match well the region of $\alpha \in(1.2,1.8)$ within which the MOM spectra for the most part are not in a parabolic shape. The same effect is visible for the more singular wing of $f(\alpha)$ spectra at $\alpha \in$ $(0.4,0.8)$. The asymmetry is even enhanced in 1991 (maximum of geomagnetic activity). The differences between more active (1991) and less active (1984) years are present in SOM spectra mainly at the wings of $f(\alpha)$ rate function as well (Figs. 12a and b). The most probable singularities around $\bar{\alpha}$ do not exhibit any changes.

\section{Discussion}

We presented an analysis of scaling and singularity characteristics of ground based and satellite magnetic fluctuations in this paper. These techniques are commonly used in studies of turbulent flows. As we outlined in the Introduction, the supposed proximity between F/SOC and MHD turbulence models may allow one to estimate measures of scaling, intermittence, etc., directly from time series, for further comparison aimed at helping the development of tractable numerical models of highly variable SW-magnetosphere interaction (Georgoulis, personal communication, 2001). We think the results are not contradictory in this sense, but rather elucidate important aspects of magnetic field fluctuations that should be incorporated into more realistic F/SOC models 
of SW driven magnetospheric activity. Scaling and singularity characteristics of high-quality geomagnetic data from THULE and NARSSARSSUAQ observatories (Single Observatory Measures) and of the $A E$-index (Multi Observatory Measure) were compared estimating PDFs, flatnesses and singularity rate functions. The same methods were applied for ACE and WIND data. The specific periods chosen for the analysis of SW fluctuations reflect the limited availability of high resolution satellite data for relatively longer periods of time. In spite of this, subgroups of disturbed and undisturbed periods were selected, having primarily in mind the occurence of an enhanced geomagnetic response represented by a 1-h mean $D_{s t}$-index. The comparison of generic features of fluctuations in SW and high-latitude SOM and MOM data is then possible, since geomagnetic activity at high-latitudes is always very high during magnetic storms, though the storm/substorm relationship itself is more complicated (Daglis et al., 2001).

It was shown that the intermittence of $A E$-index fluctuations is reduced at small scales due to the method of its derivation. We argue this also provides a possible explanation for the negligible changes in the $A E$-index singularity distribution with $\tau$. Other kinds of MOM data might be influenced in the same way. Nevertheless, the contribution of SW fluctuations makes the $A E$-index rate function asymmetric, mainly within the range of $\alpha \in(1.2,1.8)$. The same asymmetry caused by the SW is also present in THL and NAQ rate functions, however, mainly for $\tau>300 \mathrm{~min}$ (see also Vörös, 2000).

In the case of SW fluctuations it was demonstrated that the departure from Gaussian to non-Gaussian is stronger at small scales and it clearly depends on the correlation (geoeffectiveness) between IMF fluctuations and the occurence of geomagnetic storms (decreased $D_{s t}$-index). It seems to indicate that the intermittence strength of IMF magnitude and $B_{z}$ component fluctuations, in addition to other SW parameters, such as southward $B_{z}$, SW velocity, density, Alfvénic Mach number, and plasma $\beta$, represents a new parameter (or rather a whole set of parameters describing singularity features) controlling the energy input rate to the magnetosphere. Considering Taylor's hypothesis and SW velocities of $500 \mathrm{~km} / \mathrm{s}$, intermittence at time scales of tens of seconds corresponds to the spatial structures of several thousand kilometers or more. Book and Sibeck (1995) estimated the corresponding time scale on which turbulent motion may affect the transport of mass and energy across the magnetopause through interchange instability. They have found that it is less than $150 \mathrm{~s}$. As known from previous ISEE1 and 2 magnetometer studies dayside reconnection of IMF and GMF lines often seems to be a sporadic and patchy process and measurements obtained at or near the magnetopause indicate that reconnection does not necessarily occur across the whole dayside magnetopause, even under the favourable southward pointing IMF conditions (Rijnbeek et al., 1984). We conjecture that patchy reconnection may be related to intermittence and singularity characteristics of IMF turbulence at small scales. A number of works exist in which the role of turbulence in magne- topause reconnection processes is anticipated (Galeev et al., 1986; Drake et al., 1994; Kuznetsova and Roth, 1995). We recall the work of Galeev et al. (1986) in which patchy reconnection was considered to be an irregular multiscale process associated with the magnetic field diffusion and selfconsistently generated magnetic turbulence. Our results indicate that a number of singularity parameters (Hölder exponents) should be taken into account to properly describe the basic characteristics of the upstream SW turbulence. In this paper, we examined the global distribution of IMF singularities and found clear differences between geoeffectively disturbed and undisturbed periods. Obviously, to understand better the role of turbulence in patchy magnetopause reconnection processes, a proper time and space localization of IMF singularities will be needed.

As far as the magnetospheric response is considered, previous results (Vörös and Kovács, 2001) have suggested that global singularity spectra estimations of SOM and MOM data sets on different scales may allow one to separate fluctuations of SW or magnetospheric origin. Our results show that the influence of the SW is perceptible mainly at the wings of the rate function, i.e. at smaller values of $f(\alpha)$. The most probable singularities $(f(\alpha)=0.8-1)$ are less influenced by the SW driver. Rate functions estimated for years 1975-1996 exhibit similar variations as geomagnetic activity studied by Kamide et al. (1998). SW forcing effects were found when SOM and MOM singularity spectra for two years (1984 and 1991) of different geomagnetic activity levels were compared.

We believe that further development in this direction will result in a better understanding of SW-magnetosphere interaction, allowing for more efficient prediction of space weather.

Acknowledgements. The authors wish to acknowledge valuable discussions with Vincenzo Carbone, Giuseppe Consolini, Manolis Georgoulis, Alex Klimas, Nick Watkins, and Vadim Uritsky. We are grateful to Yohsuke Kamide and Ioannis Daglis for sending us their results. We acknowledge the use of the Fraclab package developed at the Institut National de Recherche en Informatique, Le Chesnay Cedex, France. Geomagnetic data from Thule observatory, Narssarssuaq observatory and $A E$-index as well as $D_{s t}$-index data from WDC Kyoto are gratefully acknowledged. We are grateful to N. Ness (Bartol Research Institute) and R. Lepping (NASA/GSFC) for making the ACE and WIND data available. Z. Vörös and D. Jankovičová were supported by VEGA grant 2/6040. P. Kovács was supported by the Hungarian Science Research Fund (OTKA) under project number F030331 and by the Eötvös Scholarship provided by the Hungarian Scholarship Committee.

\section{References}

Angelopoulos, V., Baumjohann, W., Kennel, C. F., Coroniti, F. V., Kivelson, M. G., Pellat, R., Walker, R. J., Luhr, H., and Paschmann, G.: Bursty bulk flows in the inner central plasma sheet, J. Geophys. Res, 97, 4027-4039, 1992. 
Angelopoulos, V., Mukai, T., and Kokubun, S.: Evidence for intermittency in Earth's plasma sheet and implications for selforganized criticality, Phys. Plasmas, 6, 4161-4168, 1999.

Antoni, V., Carbone, V., Cavazzana, R., Regnoli, G., Vianello, N., Spada, E., Fattorini, L., Martines, E., Serianni, G., Spolaore, M., Tramontin, L., and Veltri, P.: Transport processes in reversedfield-pinch plasmas: inconsistency with the self-organized criticality paradigm, Phys. Rev. Lett., 87, 045001-1-045001-4, 2001.

Bak, P., Tang, C., and Wiesenfeld, K.: Self-organized criticality: an explanation of 1/f noise, Phys. Rev. Lett., 33, 381-384, 1987.

Baumjohann, W., Paschmann, G., and Luhr, H.: Characteristics of high-speed ion flows in the plasma sheet, J. Geophys. Res, 95, 3801-3809, 1990.

Boffetta, G., Carbone, V., Giouliani, P., Veltri, P., and Vulpiani, A.: Power laws in solar flares: self-organized criticality or turbulence? Phys. Rev. Lett., 83, 4662-4665, 1999.

Book, D. L. and Sibeck, D. G.: Plasma transport through the magnetopause by turbulent interchange processes, J. Geophys. Res., 100, 9567-9573, 1995.

Borovsky, J. E., Elphic, R. C., Funsten, H. O., and Thomsen, M. F.: The Earth's plasma sheet as a laboratory for flow turbulence in high-bet a MHD, J. Plasma Phys., 57, 1-34, 1997.

Bruno, R., Bavassano, B., Pietropaolo, E., Carbone, V., and Veltri, P.: Effects of intermittency on interplanetary velocity and magnetic field fluctuations anisotropy, Geophys. Res. Lett., 26, 3185-3188, 1999.

Burlaga, L. F.: Intermittent turbulence in the solar wind, J. Geophys. Res, 96, 5847-5851, 1991.

Burlaga, L. F.: Multifractal structure of the magnetic field and plasma in recurrent streams at 1 AU, J. Geophys. Res, 97, 42834293, 1992.

Canus, Ch.: Robust large deviation multifractal estimation, Proc. Internat. Wavelets Conf., Tangier, 1998.

Carbone, V.: Scaling exponents of the velocity structure functions in the interplanetary medium, Ann. Geophysicae, 12, 585-590, 1994.

Chang, T.: Low-dimensional behavior and symmetry-breaking of stochastic systems near criticality: Can these effects be observed in space and in the laboratory, IEEE Trans. Plasma Sci., 20, 691694, 1992.

Chang, T.: Self-organized criticality, multi-fractal spectra, sporadic localized reconnections and intermittent turbulence in the magnetotail, Phys. Plasmas, 6, 4137-4145, 1999.

Chapman, S. C., Watkins, N. W., Dendy, R. O., Helander, P., and Rowlands, G.: A simple avalanche model as an analogue for magnetospheric activity, Geophys. Res. Lett., 25, 2397-2400, 1998.

Chapman, S. C., Dendy, R. O., and Rowlands, G.: A sandpile model with dual scaling regimes for laboratory, space and astrophysical plasmas, Phys. Plasmas, 6, 4169, 1999.

Consolini, G., Marcucci, M. F., and Candidi, M.: Multifractal structure of auroral electrojet index data, Phys. Rev. Lett., 76, 40824085, 1996.

Consolini, G.: Sandpile cellular automata and magnetospheric dynamics, in: Cosmics Physics in the Year 2000, (Eds) Aiello, S., et al., 123-126, Italy, 1997.

Consolini, G. and De Michelis, P.: Non-Gaussian distribution function of $A E$-index fluctuations: Evidence for time intermittency, Geophys. Res. Lett., 25, 4087-4090, 1998.

Consolini, G. and Lui, A. T. Y.: Sign-singularity analysis of current disruption, Geophys. Res. Lett., 26, 1673-1676, 1999.

Daglis, I. A., Baumjohann, W., Gleiss, J., Orsini, S., Sarris, T., Sc- holer, M., Tsurutani, B. T., and Vassiliadis, D.: Recent advances, open questions and future directions in Solar-Terrestrial research, Phys. Chem. Earth, 24, 5-28, 1999.

Daglis, I. A., Kozyra, J. V., Kamide, Y., Vassiliadis, D., Sharma, A. S., Liemohn, M. W., Lu, G., Gonzalez, W. D., Tsurutani, B. T., and Korth, A.: Intense space storms: 2. Critical issues and open disputes, J. Geophys. Res., submitted, 2001.

Davis, T. N. and Sugiura, M.: Auroral electrojet activity index $A E$ and its universal time variations, J. Geophys. Res, 71, 785, 1966.

Drake, J. F., Gerber, J., and Kleva, R. G.: Turbulence and transport in the magnetopause current layer, J. Geophys. Res., 99, 11211 $11223,1994$.

Dudok de Wit, T. and Krasnoselskikh, V. V.: Non-Gaussian statistics in space plasma turbulence: fractal properties and pitfalls, Nonlin. Proc. Geophys., 3, 262-273, 1996.

Freeman, M. P., Watkins, N. W., and Riley, D. J.: Evidence for a solar wind origin of the power law burst lifetime distribution of the $A E$ indices Geophys. Res. Lett., 27, 1087-1090, 2000a.

Freeman, M. P., Watkins, N. W., and Riley, D. J.: Power law burst and interburst interval distributions in the solar wind: Turbulence or dissipative SOC?, Phys. Rev. E, 62, 8794-8797, 2000b.

Galeev, A. A., Kuznetsova, M. M., and Zeleny, L. M.: Magnetopause stability threshold for patchy reconnection, Space Sci Rev., 44, 1-41, 1986.

Georgoulis, M., Kluiving, R., and Vlahos, L.: Extended instability criteria in isotropic and anisotropic energy avalanches, Physica A, 218, 191-213, 1995.

Georgoulis, M., Vilmer, N., and Crosby, N. B.: A comparison between statistical properties of solar X-ray flares and avalanche predictions in cellular automata statistical flare models, Astron. Astrophys., 367, 326-338, 2001.

Gonzalez, W. D. and Tsurutani, B. T.: Criteria of interplanetary parameters causing intense magnetic storms $\left(D_{s t}<-100 \mathrm{nT}\right)$, Planet. Space Sci., 35, 1101-1109, 1987.

Halsey, T. C., Kadanoff, J. M. H., Procaccia, L. P., and Shraiman, B. I.: Fractal measures and their singularities: the characterization of strange sets, Phys. Rev. A, 33, 1141, 1986.

Hwa, T. and Kardar, M.: Avalanches, hydrodynamics, and discharge events in models of sandpiles, Phys. Rev. A, 45, 70027023, 1992.

Isliker, H., Anastasiadis, A., Vassiliadis, D., and Vlahos, L.: Solar flare cellular automata interpreted as discretized MHD equations, Astron. Astrophys., 335, 1085-1092, 1998.

Isliker, H., Anastasiadis, A., and Vlahos, L.: MHD consistent cellular automata (CA) models I: Basic features, Astron. Astrophys. 363, 1134-1144, 2000.

Kamide, Y., Baumjohann, W., Daglis, I. A., Gonzalez, W. D., Grande, M.,Joselyn, J. A., McPherron, R. L., Phillips, J. L., Reeves, E. G. D., Rostoker, G., Sharma, A. S., Singer, H. J., Tsurutani, B. T., and Vasyliunas, V. M.: Current understanding of magnetic storms: storm/substorm relationships, J. Geophys Res., 103, 17 705-17 728, 1998.

Kamide, Y:: Geomagnetic storms as a dominant component of space weather: classic picture and recent issues, in: Space storms and space weather hazards, (Ed) Daglis, I. A., Kluwer Acad. Pub., Dordrecht, Netherlands, 43-78, 2001.

Klimas, A. J., Valdivia, J. A., Vassiliadis, D., Baker, D. N., Hesse, and Takalo, J.: Self-organized criticality in the substorm phenomenon and its relation to localized reconnection in the magnetospheric plasma sheet, J. Geophys. Res., 105, 18 765-18 780 2000.

Kovács, P. and Vörös, Z.: Geomagnetic diagnosis of the magneto- 
sphere and its dynamical interaction with the solar wind, Contr. Geophys\& Geodesy, 31, 367-374, 2001.

Kovács, P., Carbone, V., and Vörös, Z.: Wavelet-based filtering of intermittent events from geomagnetic time series, Planet. Space Sci., 49, 1219-1231, 2001.

Kuznetsova, M. M. and Roth, M.: Thresholds for magnetic percolation through the magnetopause current layer in asymmetrical magnetic fields, J. Geophys. Res., 100, 155-174, 1995.

Lu, E. T.: Avalanches in continuum driven dissipative systems, Phys. Rev. Lett., 74, 2511-2514, 1995.

Lui, A. T. Y., Chapman, S. C., Liou, K., Newell, P. T., Meng, C. I., Brittnacher, M., and Parks, G. K.: Is the dynamic magnetosphere an avalanching system?, Geophys. Res. Lett., 27, 911-914, 2000.

Marsch, E. and Tu, C. Y.: Non-Gaussian probability distributions of solar wind fluctuations, Ann. Geophysicae, 12, 1127-1138, 1994.

Marsch, E., Tu, C. Y., and Rosenbauer, H.: Multifractal scaling of the kinetic energy flux in solar wind turbulence, Ann. Geophysicae, 14, 259-269, 1996.

McPherron, R. L., Terasawa, T., and Nishida, A.: Solar wind triggering of substorm expansion onset, J. Geomagn. Geoelectr., 38, 1089-1108, 1986.

Milovanov, A. V., Zelenyi, L. M., and Zimbardo, G.: Fractal structures and power law spectra in the distant Earth's magnetotail, J. Geophys. Res., 101, 19903-19910, 1996.

Riedi, R.: An improved multifractal formalism and self-similar measures, J. Math. Anal. Appl., 189, 462-490, 1995.

Rijnbeek, R. P., Cowley, S. W. H., Southwood, D. J., and Russel, C. T.: A survey of dayside flux transfer events observed by ISEE 1 and 2 magnetometers, J. Geophys. Res., 89, 786-800, 1984.

Sharma, A. S., Sitnov, M. I., and Papadopoulos, K.: Substorms as nonequilibrioum transitions of the magnetosphere, J. Atmosph. Solar-Terr. Phys., 63, 1399-1406, 2001.

Sorriso-Valvo, L., Carbone, V., Veltri, P., Consolini, G., and Bruno, R.: Intermittency in the solar wind turbulence through probability distribution functions of fluctuations, Geophys. Res. Lett., 26, 1801-1804, 1999.

Spada, E., Carbone, V., Cavazzana, R., Fattorini, L., Regnoli, G., Vianello, N., Antoni, V., Martines, E., Serianni, G., Spolaore, M., and Tramontin, L.: Search of self-organized criticality processes in magnetically confined plasmas: hints from the reversed field pinch configuration, Phys. Rev. Lett., 86, 3032-3035, 2001.

Sugiura, M.: Hourly values of equatorial Dst for the IGY, Ann. Int. Geophys. Year, 35, 49, 1964.

Takalo, J., Timonen, J., and Koskinen, H.: Correlation dimension and affinity of $A E$ data and bicolored noise, Geophys. Res. Lett., 20, 1527-1530, 1993.

Takalo, J., Timonen, J., Klimas, A. J., Valdivia, J., and Vassiliadis, D.: Nonlinear energy dissipation in a cellular automaton magnetotail field model Geophys. Res. Lett., 26, 1813-1816, 1999.

Taylor, R. J., Lester, M., and Yeoman, T. K.: Seasonal variations in the occurence of geomagnetic storms, Ann. Geophysicae, 14,
286-289, 1996.

Tsurutani, B. T., Sugiura, M., Iyemori, T., Goldstein, B. E., Gonzalez, W. D.,Akasofu, S. I., and Smith, E. J.: The nonlinear response of $A E$ to the IMF Bs driver: A spectral break at 5 hours, Geophys. Res. Lett., 17, 279-282, 1990.

Tu, C. Y., Marsch, E., and Rosenbauer, H.: An extended structurefunction model and its application to the analysis of solar wind intermittency properties, Ann. Geophysicae, 14, 270-285, 1996.

Uritsky, V. M. and Pudovkin, M. I.: Low frequency 1/f-like fluctuations of the $A E$-index as a possible manifestation of selforganized criticality in the magnetosphere, Ann. Geophysicae, $16,1580-1588,1998$

Uritsky, V. M., Klimas, A. J., and Vassiliadis, D.: Comparative study of dynamical critical scaling in the auroral electrojet index versus solar wind fluctuations, Geophys. Res. Lett., 28, 38093812, 2001a.

Uritsky, V. M., Klimas, A. J., Valdivia, J. A., Vassiliadis, D., and Baker, D. N.: Stable critical behaviour and fast field annihilation in a magnetic field reversal model, J. Atmosph. Sol.-Terr. Physics., 63 1425-1433, $2001 b$.

Vassiliadis, D., Anastasiadis, A., Georgoulis, M., and Vlahos, L.: Derivation of solar flare cellular automata models from a subset of the magnetohydrodynamic equations, Astrophys. J., 509 L53L56, 1998.

Veltri, P. and Mangeney, A.: Scaling laws and intermittent structures in solar wind MHD turbulence, in: Solrar Wind IX, (Ed) Habbal, S., AIP Conf. Publ., in press, 1999.

Véhel, J. L.: Numerical computation of the large deviation multifractal spectrum, in CFIC96 Rome, 1996.

Véhel, J. L. and Vojak, R.: Multifractal analysis of choquet capacities: preliminary results, Adv. Appl. Math., 20, 1-43, 1998.

Vlahos, L., Georgoulis, M., Kluiving, R., and Paschos, P.: The statistical flare, Astron. Astrophys., 299, 897-911, 1995.

Vörös, Z.: Planetary indices: an attempt at synthesis, Sci. Tech. Rep., STR 98/21, GeoForschungZentrum Potsdam, 263-275, 1998.

Vörös, Z., Kovács, P., Juhász, Á., Körmendi, A., and Green, A. W.: Scaling laws from geomagnetic time series, Geophys. Res. Lett. 25, 2621-2624, 1998.

Vörös, Z.: On multifractality of high-latitude geomagnetic fluctuations, Ann. Geophysicae, 18, 1273-1282, 2000.

Vörös, Z. and Kovács, P.: Multiscale approaches in magnetospheric physics and their impact on geomagnetic data processing, Contr Geophys \& Geodesy, 31, 375-382, 2001.

Watkins, N. W., Freeman, M. P., Chapman, S. C., and Dendy, R. O.: Testing the SOC hypothesis for the magnetosphere, J. Atmosph. Sol. Terr. Phys., 63, 1435-1445, 2001 a.

Watkins, N. W., Oughton, S., and Freeman, M. P.: What can we infer about the underlying physics from burst distributions observed in an RMHD simulation?, Planet. Space Sci., 49, 1233$1237,2001 b$ 